\title{
c-Jun N-terminal kinase inhibitor favors transforming growth factor- $\beta$ to antagonize hepatitis $B$ virus $X$ protein-induced cell growth promotion in hepatocellular carcinoma
}

\author{
YAN-HUI WU, XI AI, FU-YAO LIU, HUI-FANG LIANG, BI-XIANG ZHANG and XIAO-PING CHEN \\ Hepatic Surgery Center, Tongji Hospital, Tongji Medical College, \\ Huazhong University of Science and Technology, Wuhan, Hubei 430030, P.R. China
}

Received June 5, 2014; Accepted February 2, 2015

DOI: $10.3892 / \mathrm{mmr} .2015 .4644$

\begin{abstract}
Transforming growth factor (TGF)- $\beta$ induces cell growth arrest in well-differentiated hepatocellular carcinoma (HCC) while hepatitis B virus $X$ protein $(\mathrm{HBx})$ minimizes the tumor suppression of TGF- $\beta$ signaling in early chronic hepatitis B. However, how to reverse the oncogenic effect of HBx and sustain the tumor-suppressive action of TGF- $\beta$ has yet to be investigated. The present study examined the effect of TGF- $\beta$ and a c-Jun N-terminal kinase (JNK) inhibitor on cell growth in HCC cells with forced expression of HBx. It was found that $\mathrm{HBx}$ promoted cell growth via activation of the $\mathrm{JNK} / \mathrm{pSMAD} 3 \mathrm{~L}$ pathway and inhibition of the transforming growth factor-beta type I receptor (T $\beta \mathrm{RI}) / \mathrm{pSMAD} 3 \mathrm{C}$ pathway. pSMAD3L/SMAD4 and pSMAD3C/SMAD4 complexes antagonized each other to regulate c-Myc expression. In the absence of HBx, TGF- $\beta$ induced cell growth arrest through activation of the T $\beta \mathrm{RI} / \mathrm{pSMAD} 3 \mathrm{C}$ pathway in well-differentiated HCC cells. In the presence of HBx, TGF- $\beta$ had no effect on cell growth. JNK inhibitor SP600125 significantly reversed the oncogenic action of HBx and favored TGF- $\beta$ to regain the ability to inhibit the cell growth in $\mathrm{HBx}$-expressing well-differentiated HCC cells. In conclusion, targeting JNK signaling favors TGF- $\beta$ to block $\mathrm{HBx}$-induced cell growth promotion in well-differentiated $\mathrm{HCC}$ cells. As an adjunct to anti-viral therapy, the combination of TGF- $\beta$ and inhibition of JNK signaling is a potential therapy for HBV-infected HCC.
\end{abstract}

Correspondence to: Professor Xiao-Ping Chen or Professor Bi-Xiang Zhang, Hepatic Surgery Center, Tongji Hospital, Tongji Medical College, Huazhong University of Science and Technology, 1095 Jiefang Avenue, Wuhan, Hubei 430030, P.R. China

E-mail: chenxpchenxp@163.com

E-mail: bixiangzhang@163.com

Key words: hepatitis $\mathrm{B}$ virus $\mathrm{X}$ protein, transforming growth factor- $\beta$, c-Jun N-terminal kinase inhibitor, hepatocellular carcinoma, cell growth

\section{Introduction}

Hepatocellular carcinoma (HCC) is the fifth most common cancer worldwide and one of the most fatal ones, causing nearly 600,000 mortalities yearly $(1,2)$. Hepatitis B virus (HBV) infection is one of the major causes of HCC worldwide; epidemiological studies show that $80 \%$ of all HCC occurs in $\mathrm{HBV}$-infected individuals. Hepatitis B virus $\mathrm{X}$ protein $(\mathrm{HBx})$ is a key multifunctional regulatory protein that participates in viral pathogenesis and carcinogenesis (3) and transactivates numerous transcription factors, including cyclic adenosine monophosphate response element-binding protein, activating transcription factor 2 (4), TATA-binding protein (5), activator protein 1 (AP-1) (6) and nuclear factor kappa B (7). Moreover, $\mathrm{HBx}$ has been implicated in the alteration of numerous signal transduction pathways, including the RAS/RAF/mitogen-activated protein kinase (MAPK) (8), mitogen-activated protein kinase kinase kinase 1 (MEKK1)/JNK (6), Janus kinase (JAK)/signal transducer and activator of transcription (STAT) (9), phosphoinositide 3-kinase (PI3K)/AKT (10) and Notch1 signaling (11) pathways, resulting in tumor cell growth and survival.

Transforming growth factor $\beta$ (TGF- $\beta$ ) induces cell senescence and growth arrest via the downstream SMAD signaling pathway in well-differentiated HCC cells, acting as a tumor suppressor. Activation of the TGF- $\beta$ receptor resulted in the phosphorylation of SMAD2 and SMAD3, which in turn form a hetero-trimer together with SMAD4 and translocate into the nucleus $(12,13)$. SMAD3 is a modular protein with conserved Mad-homology 1, intermediate linker and Mad-homology 2 domains (14). The C-terminal serine residue of SMAD3 is phosphorylated by activated TGF- $\beta$ type I receptor (T $\beta R I)$, whereas the linker domain is phosphorylated by other kinases, including MAPKs (15-18) and cyclin-dependent kinases (19). In contrast to the tumor-suppressive role of the C-terminal phosphorylated SMAD3 (pSMAD3C), SMAD3 phosphorylated at the linker region (pSMAD3L) correlates with enhanced cell proliferation and invasion. The different phosphorylated sites reversibly shift SMAD-dependent signaling between tumor suppression and promotion $(20,21)$. During carcinogenesis, tumor cells acquire advantage through selective reduction of the tumor-suppressive activity of TGF- $\beta$ together with 
Table I. Antibodies used in the present study.

\begin{tabular}{lll}
\hline Antigen & \multicolumn{1}{c}{ Catalog number and manufacturer } & Application \\
\hline HBx & sc-57760, Santa Cruz Biotechnology, Inc., Santa Cruz, CA, USA & $1: 200$ for WB \\
p-SMAD3C (ser423/425) & $1880-1$, Epitomics, Burlingame, CA, USA & $1: 1,000$ for WB \\
p-SMAD3L (ser213) & PA5-12694, Thermo Fisher Scientific Inc., Waltham, MA, USA & $1: 100$ for WB \\
t-SMAD3 & $1735-1$, Epitomics, Burlingame, CA, USA & $1: 2,000$ for WB \\
SMAD2/3 & sc-133098, Santa Cruz Biotechnology, Inc., Santa Cruz, CA, USA & $1: 500$ for WB \\
& & $1: 50$ for IP \\
SMAD4 & $1676-1$, Epitomics, Burlingame, CA, USA & $1: 2,000$ for WB \\
SMAD4 & sc-7154, Santa Cruz Biotechnology, Inc., Santa Cruz, CA, USA & $1: 50$ for IP \\
p-JNK (Thr183/Tyr185) & 4668, Cell Signaling Technology, Inc., Danvers, MA, USA & $1: 500$ for WB \\
t-JNK & 9258, Cell Signaling Technology, Inc., Danvers, MA, USA & $1: 1,000$ for WB \\
c-Myc & $1472-1$, Epitomics, Burlingame, CA, USA & $1: 1,000$ for WB \\
p21 & $2990-1$, Epitomics, Burlingame, CA, USA & $1: 1,000$ for WB \\
p15 & 4822, Cell Signaling Technology, Inc., Danvers, MA, USA & $1: 2,000$ for WB \\
GAPDH & KC-5G4, KangChen Bio-Tech, Shanghai, China & $1: 10,000$ for WB \\
HRP-conjugated anti-rabbit lgG & KangChen Bio-Tech, Shanghai, China & $1: 3,000$ for WB \\
HRP-conjugated anti-mouse lgG & KangChen Bio-Tech, Shanghai, China & $1: 3,000$ for WB \\
\hline
\end{tabular}

WB, western blot; IP, immunoprecipitation; p, phosphorylated; t, total; JNK, c-Jun N-terminal kinase; HBX, hepatitis B virus X protein; HRP, horseradish peroxidase.

augmentation of its oncogenic activity (22). The alterations in the TGF- $\beta$ signal transduction pathway may be involved in the development of HCC in long-standing HBV infection. In the progression of $\mathrm{HBX}$-associated hepatocarcinogenesis, HBx shifts TGF- $\beta$ signaling from the T $\beta$ RI/pSMAD3C tumor-suppressive pathway to the JNK/pSMAD3L oncogenic pathway in early chronic hepatitis B (18).

Given the roles of HBx and TGF- $\beta$, the present study hypothesized that inhibition of $\mathrm{HBx}$-induced activation of JNK/pSMAD3L sensitizes HCC cells to TGF- $\beta$ and promotes the anti-cancer activity of TGF- $\beta$. The effects of TGF- $\beta$ and JNK inhibitor SP600125 on cell growth in well-differentiated HCC cells with forced HBx expression were investigated. The present study provided an important molecular mechanism which may be utilized for the treatment of HBV-associated hepatocarcinogenesis.

\section{Materials and methods}

Cell culture. Huh-7 and Hep3B cell lines (obtained from the Liver Cancer Institute, Zhongshan Hospital, Fudan University, Shanghai, China) and the lentivirus packaging cell line 293T (purchased from China Center for Type Culture Collection, Wuhan, China) were maintained in Dulbecco's Modified Eagle's Medium (DMEM) containing 10\% fetal bovine serum (FBS; both from Hyclone, Thermo Fisher Scientific, Waltham, MA, USA), $50 \mathrm{mg} / \mathrm{ml}$ penicillin-streptomycin and $0.1 \mathrm{mM}$ non-essential amino acids (both from Hyclone, Thermo Fisher Scientific) under a humidified $5 \% \mathrm{CO}_{2}$ atmosphere at $37^{\circ} \mathrm{C}$.

Reagents and antibodies. Trypsin-EDTA was purchased from Hyclone. opti-MEM medium was from Gibco-BRL (Invitrogen Life Technologies, Carlsbad, USA). Puromycin
(Merck Calbiochem, Darmstadt, Germany), polybrene (Sigma-Aldrich, St. Louis, MO, USA), penicillin-streptomycin, Dual-Glo ${ }^{\mathrm{TM}}$ Luciferase Assay system (Promega, Madison, WI, USA), Lipofectamine 2000 with Plus (Life Technologies, Carlsbad, CA, USA) and recombinant human TGF- $\beta 1$ (carrier-free; 580706; Biolegend, San Diego, CA, USA) were used. Antibodies used in the present study are listed in Table I.

Plasmids. pLOV-cytomegalovirus (CMV)-enhanced green fluorescent protein (eGFP)-HBx and pLOV-CMV-eGFP were constructed by NeuronBiotech (Shanghai, China). Lentiviral gene expression vector GV266-vec, lentiviral packaging vector pHelper 1.0 and pHelper 2.0 were purchased from GeneChem Co, Ltd, (Shanghai, China). The luciferase reporter assay driven by Smad binding element 4 (SBE4-luc) SBE4-luc (Addgene plasmid no. 16495) was a gift from Professor Bert Vogelstein (Department of Pathology, Kimmel Cancer Center, Johns Hopkins University, Baltimore, USA) (23). pRL-TK was purchased from Promega (Madison, WI, USA).

Lentivirus production, viral infection and establishment of stable clones. Lentiviral supernatants were produced as described previously (24). Briefly, 293T cells were transfected with pLOV-CMV-eGFP-HBx or pLOV-CMV-eGFP plasmid by Lipofectamine 2000 and Plus, which were diluted with opti-MEM (Gibco Life Technologies, Carlsbad, CA, USA), then selected by $5 \mu \mathrm{g} / \mathrm{ml}$ puromycin for 2 weeks and finally used to collect lentivirus-containing supernatants. The collected retroviral and lentiviral supernatants were filtered through a $0.45-\mu \mathrm{m}$ filter (PALL, Port Washington, NY, USA), concentrated by Centricon Plus 70 (Merck 
A

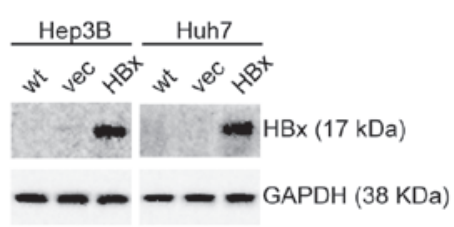

C

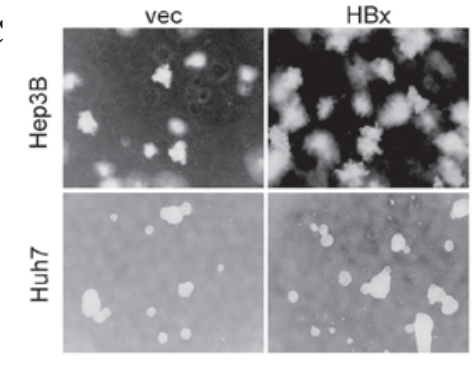

B

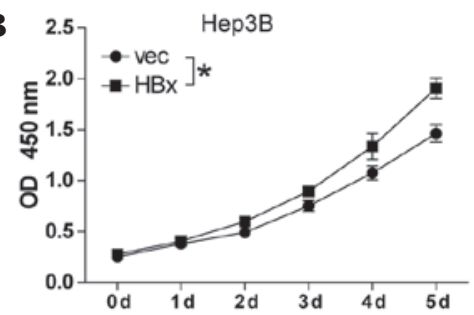

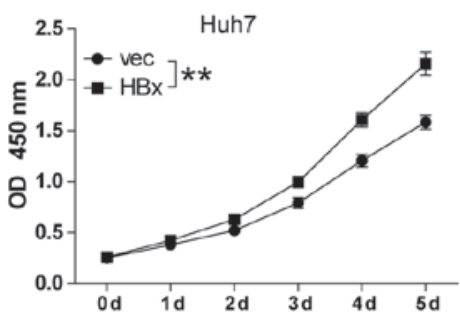

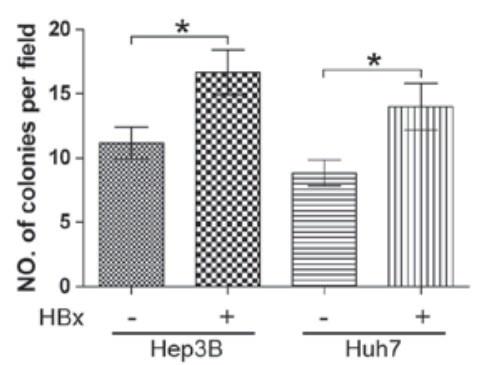

Figure 1. HBx promotes cell growth in well-differentiated hepatocellular carcinoma cell lines. (A) Expression of HBx in Hep3B and Huh7 stable clones generated by lentiviral pLOV-CMV-eGFP vector or pLOV-CMV-eGFP-HBx transfection. (B) A cell counting kit 8 cell proliferation assay was performed. Cells (1.5x10 $\left.{ }^{3}\right)$ were cultured with complete culture medium for five days. All experiments were conducted in triplicate. ${ }^{*} \mathrm{P}<0.05$, ${ }^{* *} \mathrm{P}<0.01$. (C) Soft agarose assay was performed. Cells $\left(1 \times 10^{4} / \mathrm{ml}\right)$ were cultured for two weeks. Colonies grown on soft agarose were counted and pictures of colonies were captured (magnification, $\left.\mathrm{x} 100\right)$. Values are expressed as the mean \pm standard error of the mean of six fields, with experiments conducted in triplicate. ${ }^{*} \mathrm{P}<0.05$, ${ }^{* *} \mathrm{P}<0.01$. HBx, hepatitis $\mathrm{B}$ virus $\mathrm{X}$ protein; eGFP, enhanced green fluorescent protein; CMV, cytomegalovirus; vec, vector; OD, optical density; wt, wild-type.

Millipore, Darmstadt, Germany) according to the manufacturer's instructions and used for infection of HCC cells. $72 \mathrm{~h}$ following infection, cells were selected with $2 \mu \mathrm{g} / \mathrm{ml}$ and $3 \mu \mathrm{g} / \mathrm{ml}$ puromycin for Hep3B and Huh7 cells, respectively for 2 weeks. Selected pools of cells were used for the subsequent experiments.

Cell proliferation assay. Cell proliferation was measured using the Cell Counting Kit-8 (Beyotime Institute of Biotechnology, Shanghai, China) according to the manufacturer's instructions. Hep3B and Huh7 $\left(1.5 \times 10^{3}\right)$ cells were treated with TGF- $\beta 1(10 \mathrm{ng} / \mathrm{ml})$ or SP600125 $(10 \mu \mathrm{M})$, which was replaced every $48 \mathrm{~h}$ for a total of five days. At the indicated time-points, cells were incubated with CCK-8 stain for $2 \mathrm{~h}$ according to the manufacturer's instructions. The cell proliferation rate was assessed by measuring the absorbance at $450 \mathrm{~nm}$ using a microplate reader (Elx 800; BioTek Instruments, Inc., Winooski, VT, USA).

Soft agarose tumorigenicity assay. The soft agarose assay was performed as described previously (11). Briefly, Hep3B and Huh7 cells $\left(1 \times 10^{4}\right)$ were suspended in $1 \mathrm{ml} 0.4 \%$ sea plaque agarose (A9045; Sigma-Aldrich) containing 10\% FBS and then plated on top of $1 \mathrm{ml}$ semisolid $0.8 \%$ agarose in six-well plates. Cells were treated with TGF- $\beta 1(10 \mathrm{ng} / \mathrm{ml})$ or SP600125 $(10 \mu \mathrm{M})$ very $48 \mathrm{~h}$ for two weeks. Colonies grown on soft agarose were counted and pictures of colonies were captured using a magnification of 100x using a phase-contrast microscope (Eclipse C1 System, Nikon, Tokyo, Japan).

Co-immunoprecipitation (co-IP) and western blot analyses. Cells were lysed by sonication in radioimmunoprecipitation assay lysis buffer (P0013D; Beyotime Institute of Biotechnology) supplemented with Protease Inhibitor Cocktail and Phosphatase Inhibitor PhosSTOP Tablets (Roche
Applied Science, Basel, Switzerland). Protein content was then measured with the Bicinchoninic Acid kit (Beyotime Institute of Biotechnology). The co-IP assays were performed as previous described $(25,26)$ with certain modifications. Specifically, $\sim 1 \times 10^{7}$ cells were lysed by sonication in $1 \mathrm{ml} \mathrm{NP}-40$ lysis buffer (P0013F; Beyotime Institute of Biotechnology) supplemented with the Protease Inhibitor Cocktail and PhosSTOP Cocktail Tablets. Lysates were pre-cleared with purified mouse or rabbit IgG (Wuhan Boster Biological Technology, Ltd., Wuhan, China) antibodies for $15 \mathrm{~min}$ at $4^{\circ} \mathrm{C}$ on a rotating platform, then were centrifuged at $15,000 \mathrm{x} g$ for $15 \mathrm{~min}$. Supernatants were incubated with the corresponding antibodies overnight, then were incubated with protein A/G beads (Abmart, Inc., Shanghai, China) for $4 \mathrm{~h}$ at $4^{\circ} \mathrm{C}$ on a rotating platform. Immunoprecipitates (IPs) and equal quantities of cell lysates $(40 \mu \mathrm{g})$ were then subjected to western blot analyses. Samples of extract taken prior to IP were processed in parallel with the IPs and were considered as the "inputs". Cell lysates or immunoprecipitates were separated with SDS-PAGE (Wuhan Goodbio Technology Co., Ltd., Wuhan, China) and transferred to polyvinylidene difluoride membranes (Roche Applied Science). Nonspecific binding was blocked with Tris-buffered saline (TBS) containing 5\% non-fatty milk (Inner Mongolia Yili Industrial Group Limited by Share Ltd, Hohhot, China) or bovine serum albumin (Roche Applied Science) by incubating the membranes for $1 \mathrm{~h}$ at $37^{\circ} \mathrm{C}$. The blots were then probed overnight at $4^{\circ} \mathrm{C}$ on a rotating platform with primary antibodies in TBS with $0.1 \%$ Tween 20 (Sigma-Aldrich). Membranes were then washed three times with TBS and incubated with horseradish peroxidase-conjugated secondary antibodies (Jackson ImmunoResearch Laboratories, Inc., West Grove, PA, USA). Immunoreactive bands were detected with chemiluminescence (Life Technologies) and luminous signals from reactive bands were assessed with the Alpha Innotech Fluorochem Imaging system (Alphatron Asia Pte Ltd, Singapore, Singapore). 

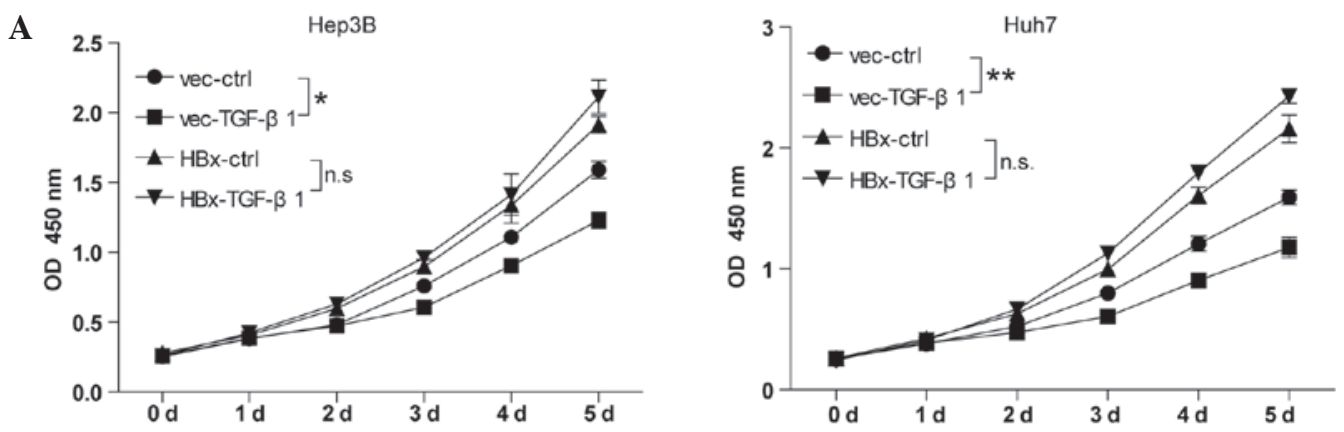

B
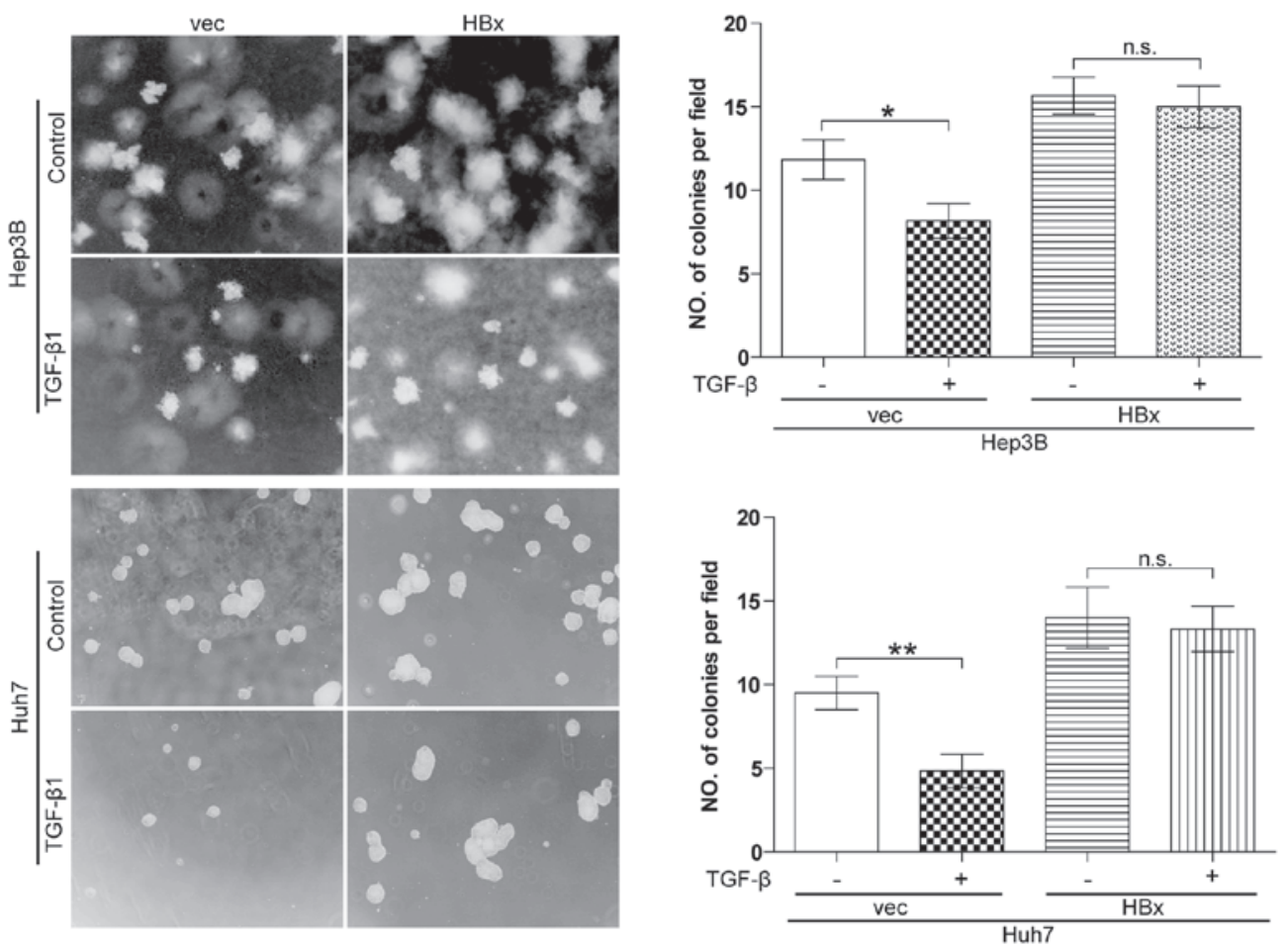

Figure 2. TGF- $\beta$ has no effect on cell growth in well-differentiated hepatocellular carcinoma cell lines with forced HBx expression. (A) A cell counting kit 8 cell proliferation assay was performed. Cells were treated with $10 \mathrm{ng} / \mathrm{ml}$ TGF- $\beta 1$ every $48 \mathrm{~h}$ for five days. All experiments were conducted in triplicate. ${ }^{*} \mathrm{P}<0.05,{ }^{* *} \mathrm{P}<0.01$. (B) A soft agarose assay was performed. Cells were treated with $10 \mathrm{ng} / \mathrm{ml}$ TGF- $\beta 1$ every $48 \mathrm{~h}$ for two weeks. Values are expressed as the mean \pm standard error of the mean of six fields, with experiments conducted in triplicate. Magnification, $\mathrm{x} 100 .{ }^{*} \mathrm{P}<0.05,{ }^{* *} \mathrm{P}<0.01$. TGF, transforming growth factor; HBx, hepatitis B virus X protein; vec, vector; ctrl, control; OD, optical density; wt, wild-type.

Transcriptional response assay. A transcriptional response assay was performed as described previously (13). Briefly, cells seeded in a 24-well plate were transiently co-transfected with SBE4-Luc together with p-RL-TK reporter constructs. Cells were treated with TGF- $\beta(10 \mathrm{ng} / \mathrm{ml})$ for $24 \mathrm{~h}(24 \mathrm{~h}$ after transfection) and then luciferase activity was detected using the Dual-Luciferase Reporter Assay System (Promega) according to the manufacturer's instructions. The relative luciferase activity was determined by a GloMax 20/20 Luminometer (Promega). Luciferase activity was normalized to Renilla activity and presented as the mean \pm standard error of the mean (SEM) of triplicate measurements.

Statistical analysis. Values are expressed as the mean \pm SEM of triplicate experiments. Statistical analyses were performed using SPSS 13.0 (SPSS, Inc., Chicago, IL, USA) software. One-way analysis of variance or Student's $t$-test were used for analyzing quantitative data between two groups. $\mathrm{P}<0.05$ was considered to indicate a statistically significant difference between values.

\section{Results}

HBx promotes cell growth in well-differentiated HCC cell lines. HBx has been reported to be implicated in hepatocarcinogenesis (3) and the present study was the first, to the best of our knowledge, to investigate the effect of HBx on HCC cell growth. The well-differentiated HCC cell lines Hep3B and Huh7 cells were selected to establish stable clones with either forced expression of HBx (Hep3B-HBx and Huh7-HBx) or which were transfected with the empty vector as a negative control (Hep3B-vec and Huh7-vec). Stable clones were confirmed by western blot analysis (Fig. 1A). The effects of altered expression of HBx on cell growth were then assessed using the CCK8 cell proliferation assay. It was found that forced expression of HBx resulted in an increase in the cell proliferation of Hep3B $(\mathrm{P}<0.05)$ and Huh7 cells $(\mathrm{P}<0.01)$ (Fig. 1B). In addition, a soft agarose assay was conducted, and a significant enhancement of Hep3B cell growth $(\mathrm{P}<0.05)$ and Huh7 cell growth $(\mathrm{P}<0.05)$ was observed (Fig. 1C). 


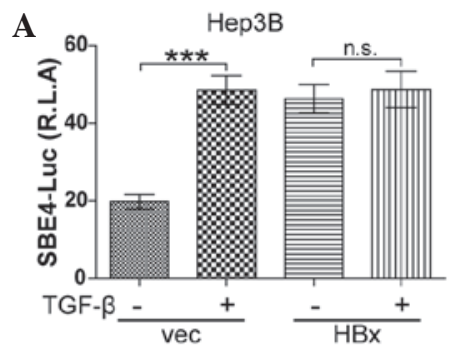

B
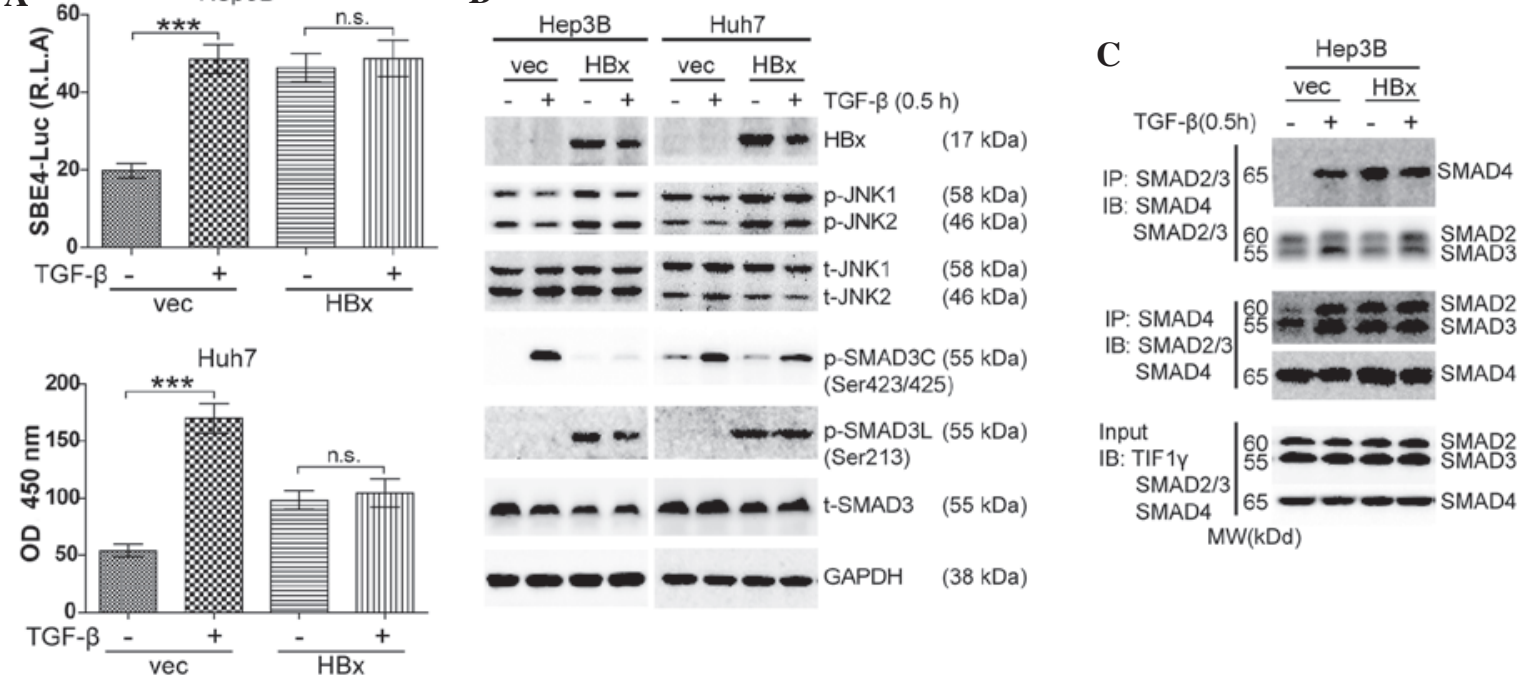

Figure 3. HBx activates JNK/pSMAD3L signaling and promotes pSMAD3L/SMAD4 complex formation. (A) Hep3B and Huh7 cells were co-transfected with SBE4-luc and pRL-TK for $24 \mathrm{~h}$ and then treated with TGF- $\beta 1(10 \mathrm{ng} / \mathrm{ml})$ for $24 \mathrm{~h}$. Luciferase activity was normalized to renilla luciferase activity and expressed as the mean \pm standard error of the mean of triplicate measurements. ${ }^{* * *} \mathrm{P}<0.001$. (B) Lysates from Hep3B and Huh7 cells treated with $10 \mathrm{ng} / \mathrm{ml}$ TGF- $\beta 1$ for $0.5 \mathrm{~h}$ were subjected to western blot analysis to examine the indicated proteins. (C) Lysates from Hep3B cells treated with $10 \mathrm{ng} / \mathrm{ml}$ TGF- $\beta 1$ for $0.5 \mathrm{~h}$ were subjected to IP of SMAD2/3 or SMAD4 followed by western blotting with SMAD4 or SMAD2/3 antibody. Input lysates were used for western blotting with antibodies against indicated proteins. All experiments were conducted in triplicate. TGF, transforming growth factor; Luc, luciferase; OD, optical density; vec, vector; p, phosphorylated; t, total; JNK, c-Jun N-terminal kinase; HBX, hepatitis B virus X protein; IP, immunoprecipitation; IB, immunoblot.
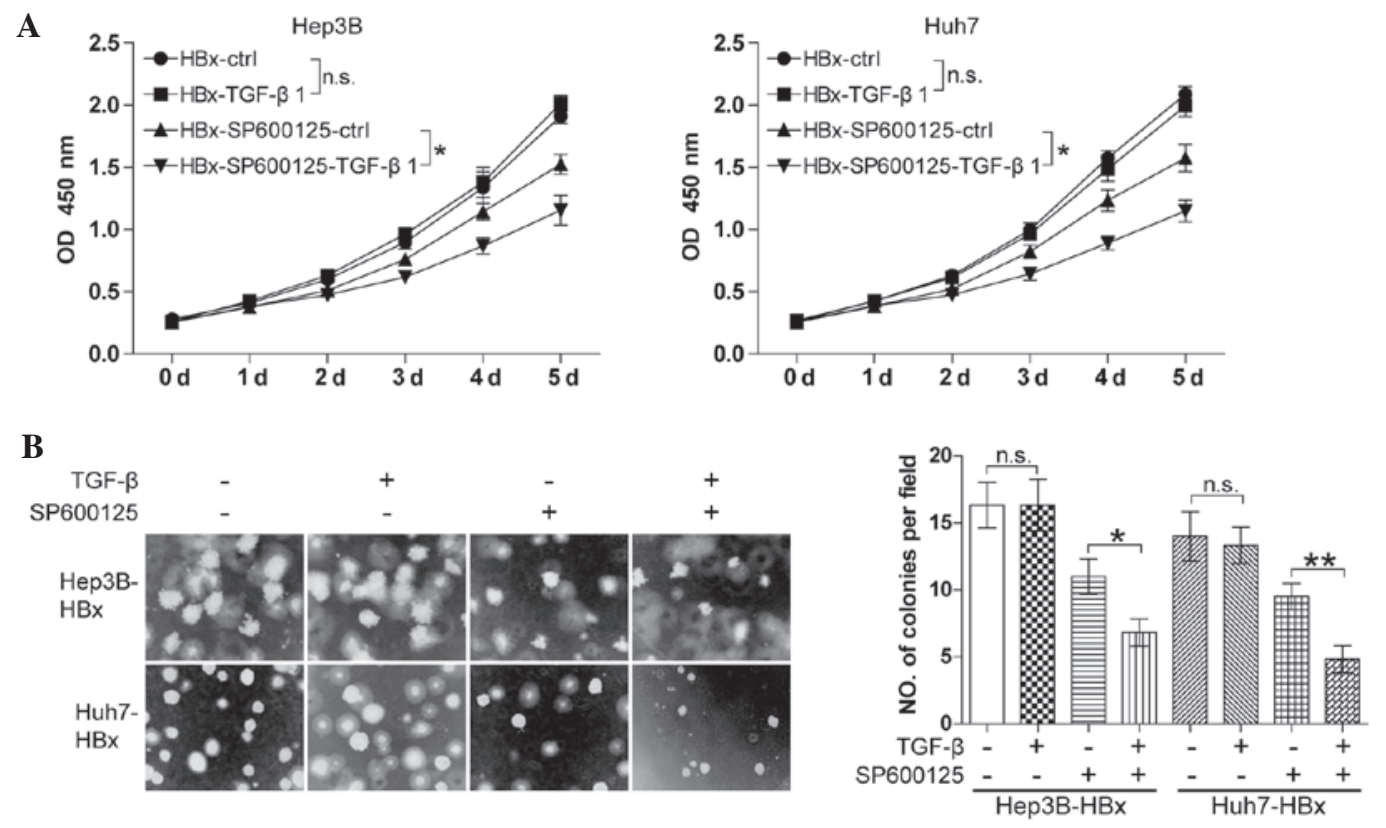

Figure 4. Targeting the c-Jun N-terminal kinase pathway favors TGF- $\beta$ to antagonize the oncogenic action of HBx. (A) A cell counting kit 8 cell proliferation assay was performed. Cells were treated with $10 \mathrm{ng} / \mathrm{ml}$ TGF- $\beta 1$ together with DMSO or $10 \mu \mathrm{M} \mathrm{SP} 600125$ every $48 \mathrm{~h}$ for five days. All experiments were conducted in triplicate. ${ }^{*} \mathrm{P}<0.05,{ }^{* *} \mathrm{P}<0.01$. (B) A soft agarose assay was performed. Cells were treated with $10 \mathrm{ng} / \mathrm{ml}$ TGF- $\beta 1$ together with DMSO or $10 \mu \mathrm{M}$ SP600125 every $48 \mathrm{~h}$ for two weeks. Values are expressed as the mean \pm standard error of the mean of results from triplicate experiments. Magnification, $\mathrm{x} 100$. ${ }^{*} \mathrm{P}<0.05,{ }^{* *} \mathrm{P}<0.01$. TGF, transforming growth factor; HBx, hepatitis B virus X protein; vec, vector; ctrl, control; OD, optical density; DMSO, dimethylsulfoxide.

TGF- $\beta$ has no effect on cell growth in well-differentiated $H C C$ cell lines with forced HBx expression. Autocrine TGF- $\beta$ signaling is commonly observed in HCC cells and TGF- $\beta$ signaling has a tumor-suppressive role in the initial stage of HCC (13). However, the effect of TGF- $\beta$ signaling in HBx-expressing HCC cells had not been elucidated thus far. The present study detected the effect of TGF- $\beta$ on cell growth in vector control cells (Hep3B-vec and Huh7-vec) and cells with forced $\mathrm{HBx}$ expression (Hep3B-HBx and Huh7-HBx) using the CCK8 cell proliferation assay. In the absence of HBx, TGF- $\beta$ significantly inhibited cell growth in Hep3B and Huh7 cell lines. By contrast, in the presence of HBx, Hep3B and Huh7 cells were significantly resistant to TGF- $\beta$-induced growth arrest (Fig. 2A). The soft agarose assay (Fig. 2B) produced similar results. These results indicated that TGF- $\beta$-induced cell growth arrest was blocked by HBx. 
A

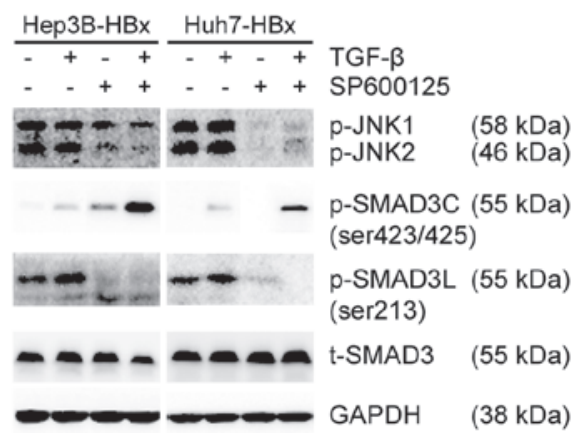

B

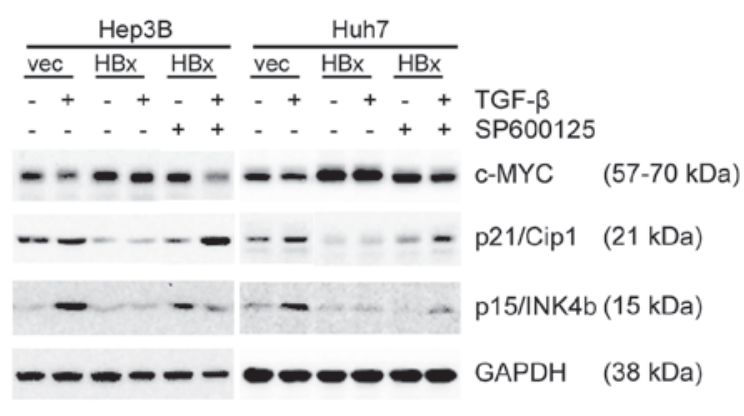

Figure 5. JNK inhibitor SP600125 promotes pSMAD3C/SMAD4 complex-induced reduction of c-Myc expression. (A) Lysates from Hep3B-HBx and Huh7-HBx cells treated with $10 \mathrm{ng} / \mathrm{ml}$ TGF- $\beta 1$ together with DMSO or $10 \mu \mathrm{M} \mathrm{SP} 600125$ for $0.5 \mathrm{~h}$ were subjected to western blot with p-JNK1/2, pSMAD3C (Ser423/425) and pSMAD3L (Ser213). (B) Lysates from Hep3B and Huh7 cells treated with $10 \mathrm{ng} / \mathrm{ml}$ TGF- $\beta 1$ together with DMSO or $10 \mu$ M SP600125 for $72 \mathrm{~h}$ were subjected to western blot analysis with c-Myc, p21/Cip1 and p15/INK4b. JNK, c-Jun N-terminal kinase; vec, vector; p, phosphorylated; t, total; HBX, hepatitis B virus X protein; DMSO, dimethyl sulfoxide.

HBX activates JNK/pSMAD3L signaling and promotes pSMAD3L/SMAD4 complex formation. To explore the mechanism by which HBx blocked TGF- $\beta 1$-induced cell growth arrest in well-differentiated HCC cells, the present study detected the TGF- $\beta /$ SMAD signaling activity in response to exogenous TGF- $\beta 1$ in the absence or presence of $\mathrm{HBx}$ with a luciferase reporter assay using SBE4-luc. It was observed that Hep3B and Huh7 displayed intact TGF- $\beta$ signaling activity in the absence of HBx. Forced expression of HBx enhanced SMAD signaling, which was not affected by TGF- $\beta$ (Fig. 3A). Next, western blot analysis was performed to evaluate the activation of SMAD signaling. In the absence of HBx, TGF- $\beta$ predominantly phosphorylated SMAD3 at the $\mathrm{C}$-terminal region (pSMAD3C). In the presence of $\mathrm{HBx}$, elevated JNK phosphorylation (p-JNK) was found followed by elevated pSMAD3L together with reduced pSMAD3C levels. The elevated p-JNK/pSMAD3L was independent of TGF- $\beta$ (Fig. 3B). Finally, immunoprecipitation followed by western blotting was used to examine the formation of the SMAD2/3/4 complex. In Hep3B cells, TGF- $\beta$ induced SMAD2/3/4 complex formation in the absence of HBx. Forced expression of HBx resulted in an increased formation of SMAD2/3/4 complex, which was not enhanced by TGF- $\beta$ (Fig. $3 C$ ). In conclusion, TGF- $\beta$-induced cell growth arrest was blocked by HBx via activation of JNK/pSMAD3L signaling and an increase in pSMAD3L/SMAD4 complex formation.

Targeting the JNK pathway favors TGF- $\beta$ to antagonize the oncogenic action of $H B x$. Elevated JNK phosphorylation induced by HBx is known to be essential for the phosphorylation of SMAD3 at the linker region for the restriction of the tumor-suppressive action of TGF- $\beta$ (18). The present study hypothesized that inhibition of the JNK pathway may restore the ability of TGF- $\beta$ to induce cell growth arrest in HBx-expressing HCC cells. To confirm this, the Hep3B-HBX and Huh7-HBX cell lines were treated with TGF- $\beta$ together with or without JNK inhibitor SP600125. The CCK8 cell proliferation assay showed that TGF- $\beta$ had no significant effect on cell growth in the absence of SP600125. SP600125 significantly inhibited the cell growth and the combination of SP600125 and TGF- $\beta$ resulted in an increased reduction in cell growth compared with that of SP600125 alone (Fig. 4A). In addition, a similar result was observed using the soft agarose assay (Fig. 4B). The results showed that JNK inhibitor SP600125 restored the capacity of TGF- $\beta$ to induce cell growth arrest in HBx-expressing well-differentiated HCC cells.

JNK inhibitor SP600125 promotes pSMAD3C/SMAD4 complexe-induced reduction of c-Myc expression. To explore the underlying mechanism by which JNK inhibitor SP600125 favored TGF- $\beta$ to antagonize the oncogenic action of HBx, the phosphorylation levels of JNK and SMAD3 were examined. SP600125 significantly inhibited the phosphorylation of JNK and SMAD3L and promoted the phosphorylation of SMAD3C induced by TGF- $\beta$ (Fig. 5A). c-Myc is implicated in HCC cell senescence and cell cycle progression, and its targeting of the SMAD2/3/4 transcription complex leads to the inhibition of p15 and p21 $(13,27)$. Therefore, the present study assessed the protein expression of c-Myc, p21 and p15 by western blot analysis. In the absence of HBx, TGF- $\beta$ induced reduction of c-Myc expression followed by upregulation of p21 and p15 expression. By contrast, forced expression of HBx resulted in an opposite effect. In the presence of $\mathrm{HBx}$, inhibition of JNK/Psmad3L signaling with SP600125 resulted in reduced $\mathrm{c}-\mathrm{Myc}$ expression and upregulation of $\mathrm{p} 21$ and $\mathrm{p} 15$ expression, while TGF- $\beta$ had no significant effect on c-Myc, p15 and p21 expression. In combination with SP600125, TGF- $\beta$ regained the ability to inhibit c-Myc expression followed by the upregulation of $\mathrm{p} 21$ and $\mathrm{p} 15$ expression (Fig. 5B). In conclusion, pSMAD3L/SMAD4 and pSMAD3C/SMAD4 complexes antagonize each other to regulate c-Myc expression. SP600125 significantly inhibited the phosphorylation of JNK and SMAD3L and promoted pSMAD3C/SMAD4 complex-induced reduction of c-Myc expression.

\section{Discussion}

$\mathrm{HBx}$ has a major role in the association between chronic HBV infection and the development of HCC (28). HBx acts as a transactivator to activate numerous key pathways dependent on various cell contexts, including the RAS/RAF/MAPK (8), MEKK1/JNK (6), JAK/STAT (9), PI3K/AKT (10) and Notch1 signaling (11) pathways, resulting in tumor cell growth 
and survival. HBx also acts as a transcriptional factor that stimulates the expression of proto-oncogenes, which control hepatocellular proliferation, transformation, apoptosis and DNA repair (29). In the present study, it was found that HBx promoted cell growth via activation of the JNK/pSMAD3L pathway in well-differentiated HCC cell lines, which was consistent with the results of previous studies $(10,18)$. Even though HBx may transactivate certain other signaling pathways, demonstration of the activation of the JNK/pSMAD3L pathway sufficiently illustrates the oncogenic action of $\mathrm{HBx}$.

Autocrine TGF- $\beta$ is commonly observed in HCC cells and its expression pattern is closely correlated with SA- $\beta-\mathrm{Gal}$ activities in normal liver, cirrhosis and HCC (13). These findings indicated that TGF- $\beta$ may be a crucial factor to induce cell senescence and restrain the progression of HCC in its initial stages. Combined with its potential to induce growth arrest and apoptosis $(13,30)$, TGF- $\beta$ treatment may be an attractive therapeutic option for the prevention of HCC. However, this strategy is counteracted by fully established HCC tumor cells, which reverse the actions of TGF- $\beta$ with the help of other factors (31). In the present study, HBx was identified to be one such factor. In the absence of $\mathrm{HBx}$, TGF- $\beta$ induced cell growth arrest in well-differentiated HCC cell lines. By contrast, TGF- $\beta$ had no effect on cell growth in the presence of $\mathrm{HBx}$. This result, together with those of previous studies $(10,18)$, indicated that blocking the transaction of $\mathrm{HBx}$ is an effective method to restore the tumor-suppressive function of TGF- $\beta$. In other words, inhibition of JNK/pSMAD3L signaling may maintain the tumor-suppressive action of TGF- $\beta$. The present study found that a JNK inhibitor, SP600125, significantly inhibited the cell growth in well-differentiated HCC cell lines. In the presence of SP600125, TGF- $\beta$ regained its ability to induce cell growth arrest in HCC cell lines with forced expression of HBx. These results suggested that targeting JNK/pSMAD3L signaling is a potential therapy for $\mathrm{HBV}$-infected patients with HCC.

TGF- $\beta /$ SMAD signaling regulates transcription of numerous targeted genes, including plasminogen activator inhibitor 1 (31) and c-Jun (32). In this process, numerous co-activators, including Cited2 (33), nuclear factor of activated T-cells (NFAT) (34), AP-1 (32) and CREB binding protein/P300 (35), or co-suppressors, including c-Ski, SnoN (36) and mSin3A (37), have a key role. The profile of SMAD-binding cofactors during development or under various growth conditions determines cellular responses to TGF- $\beta$. The proto-oncogene $c-M y c$ is one target of TGF- $\beta /$ SMAD signaling. The transcriptional repression of c-Myc is dependent on direct SMAD3 binding to a novel SMAD binding site, termed a repressive SMAD binding element, within the TGF- $\beta$ inhibitory element of the c-Myc promoter (38). Consistent with a previous study (38), it was shown that the pSMAD3C/SMAD4 transcriptional complex reduced the expression of c-Myc. In addition, the pSMAD3L/SMAD4 transcriptional complex had an opposite effect. pSMAD3C/SMAD4 complex recruits co-suppressors, including p107, to the c-Myc promoter, inhibiting the transcription of c-Myc (38). These results indicated that the pSMAD3L/SMAD4 transcriptional complex promoted c-Myc transcription via recruitment of co-activators of c-Myc transcription, including Cited2 (33) and NFAT (34).
In conclusion, the present study revealed a dual role of TGF- $\beta$ on cell growth in well-differentiated HCC, which is dependent on HBx. HBx shifts TGF- $\beta$ from a tumor suppressor to a tumor promoter. Therefore, anti-HBV therapy is very important to improve HCC prognosis. Besides anti-viral therapy, inhibition of activated JNK/pSMAD3L signaling caused by HBx is an important and potential therapy for HBV-infected patients with HCC.

\section{Acknowledgements}

The present study was supported by grants from the National Natural Science Foundation of China (nos. 81372327 and 81072001), the State Key Project on Infection Disease of China (nos. 2012ZX10002016-004 and 2012ZX10002010-001-004), and the Chinese Ministry of Public Health for Key Clinical Project [no. (2010) 493-51] to XP Chen. The authors are very grateful to Dr Arian Laurence (Molecular Immunology and Inflammation Branch, National Institute of Arthritis and Musculoskeletal and Skin Diseases, National Institutes of Health, Bethesda, MD, USA) for revising the manuscript.

\section{References}

1. Llovet JM, Burroughs A and Bruix J: Hepatocellular carcinoma. Lancet 362: 1907-1917, 2003.

2. Aravalli RN, Steer CJ and Cressman EN: Molecular mechanisms of hepatocellular carcinoma. Hepatology 48: 2047-2063, 2008.

3. Bouchard MJ and Schneider RJ: The enigmatic X gene of hepatitis B virus. J Virol 78: 12725-12734, 2004.

4. Maguire HF, Hoeffler JP and Siddiqui A: HBV X protein alters the DNA binding specificity of CREB and ATF-2 by protein-protein interactions. Science 252: 842-844, 1991.

5. Qadri I, Maguire HF and Siddiqui A: Hepatitis B virus transactivator protein $\mathrm{X}$ interacts with the TATA-binding protein. Proc Natl Acad Sci USA 92: 1003-1007, 1995.

6. Benn J, Su F, Doria M and Schneider RJ: Hepatitis B virus HBx protein induces transcription factor AP-1 by activation of extracellular signal-regulated and c-Jun N-terminal mitogen-activated protein kinases. J Virol 70: 4978-4985, 1996.

7. $\mathrm{Su} \mathrm{F}$ and Schneider RJ: Hepatitis B virus HBx protein activates transcription factor NF-kappaB by acting on multiple cytoplasmic inhibitors of rel-related proteins. J Virol 70: 4558-4566, 1996.

8. Benn J and Schneider RJ: Hepatitis B virus HBx protein activates Ras-GTP complex formation and establishes a Ras, Raf, MAP kinase signaling cascade. Proc Natl Acad Sci USA 91: 10350-10354, 1994.

9. Lee YH and Yun Y: HBx protein of hepatitis B virus activates Jak1-STAT signaling. J Biol Chem 273: 25510-25515, 1998.

10. Shih WL, Kuo ML, Chuang SE, Cheng AL and Doong SL: Hepatitis $B$ virus $X$ protein inhibits transforming growth factor- $\beta$-induced apoptosis through the activation of phosphatidylinositol 3-kinase pathway. J Biol Chem 275: 25858-25864, 2000.

11. Xu J, Yun X, Jiang J, et al: Hepatitis B virus X protein blunts senescence-like growth arrest of human hepatocellular carcinoma by reducing Notch1 cleavage. Hepatology 52: 142-154, 2010.

12. Heldin $\mathrm{CH}$, Miyazono $\mathrm{K}$ and ten Dijke P: TGF-beta signalling from cell membrane to nucleus through SMAD proteins. Nature 390: 465-471, 1997.

13. Senturk S, Mumcuoglu M, Gursoy-Yuzugullu O, Cingoz B, Akcali KC and Ozturk M: Transforming growth factor-beta induces senescence in hepatocellular carcinoma cells and inhibits tumor growth. Hepatology 52: 966-974, 2010.

14. Massagué J: TGF-beta signal transduction. Ann Rev Biochem 67: 753-791, 1998.

15. Kretzschmar M, Doody J, Timokhina I and Massagué J: A mechanism of repression of TGFbeta/Smad signaling by oncogenic Ras. Genes Dev 13: 804-816, 1999.

16. Mori S, Matsuzaki K, Yoshida K, et al: TGF-beta and HGF transmit the signals through JNK-dependent Smad2/3 phosphorylation at the linker regions. Oncogene 23: 7416-7429, 2004. 
17. Furukawa F, Matsuzaki K, Mori S, et al: p38 MAPK mediates fibrogenic signal through Smad3 phosphorylation in rat myofibroblasts. Hepatology 38: 879-889, 2003.

18. Murata M, Matsuzaki K, Yoshida K, et al: Hepatitis B virus $X$ protein shifts human hepatic transforming growth factor (TGF)-beta signaling from tumor suppression to oncogenesis in early chronic hepatitis B. Hepatology 49: 1203-1217, 2009.

19. Matsuura I, Denissova NG, Wang G, He D, Long J and Liu F: Cyclin-dependent kinases regulate the antiproliferative function of Smads. Nature 430: 226-231, 2004.

20. Yamagata H, Matsuzaki K, Mori S, et al: Acceleration of Smad2 and Smad3 phosphorylation via c-Jun NH(2)-terminal kinase during human colorectal carcinogenesis. Cancer Res 65: 157-165, 2005.

21. Sekimoto G, Matsuzaki K, Yoshida K, et al: Reversible Smad-dependent signaling between tumor suppression and oncogenesis. Cancer Res 67: 5090-5096, 2007.

22. Roberts AB and Sporn MB: The Transforming Growth Factor- $\beta$ s. In: Peptide Growth Factors And Their Receptors. Springer: pp419-472, 1990.

23. Zawel L, Dai JL, Buckhaults P, et al: Human Smad3 and Smad4 are sequence-specific transcription activators. Mol Cell 1: 611-617, 1998.

24. Ding ZY, Jin GN, Liang HF, et al: Transforming growth factor $\beta$ induces expression of connective tissue growth factor in hepatic progenitor cells through Smad independent signalling. Cell Signal 25: 1981-1992, 2013.

25. Xue J, Lin X, Chiu WT, et al: Sustained activation of SMAD3/SMAD4 by FOXM1 promotes TGF- $\beta$-dependent cancer metastasis. J Clin Invest 124: 564-579, 2014.

26. Dupont S, Mamidi A, Cordenonsi M, et al: FAM/USP9x, a deubiquitinating enzyme essential for TGFbeta signaling, controls Smad4 monoubiquitination. Cell 136: 123-135, 2009.

27. Siegel PM and Massagué J: Cytostatic and apoptotic actions of TGF- $\beta$ in homeostasis and cancer. Nat Rev Cancer 3: 807-821, 2003.

28. Block TM, Mehta AS, Fimmel CJ and Jordan R: Molecular viral oncology of hepatocellular carcinoma. Oncogene 22: 5093-5107, 2003.
29. Zhang X, Zhang H and Ye L: Effects of hepatitis B virus X protein on the development of liver cancer. J Lab Clin Med 147: 58-66, 2006

30. Dzieran J, Fabian J, Feng T, et al: Comparative analysis of TGF- $\beta /$ Smad signaling dependent cytostasis in human hepatocellular carcinoma cell lines. PloS one 8: e72252, 2013.

31. Dennler S, Itoh S, Vivien D, ten Dijke P, Huet S and Gauthier JM: Direct binding of Smad3 and Smad4 to critical TGF $\beta$-inducible elements in the promoter of human plasminogen activator inhibitor-type 1 gene. EMBO J 17: 3091-3100, 1998.

32. Wong C, Rougier-Chapman EM, Frederick JP, et al: Smad3-Smad4 and AP-1 complexes synergize in transcriptional activation of the c-Jun promoter by transforming growth factor beta. Mol Cell Biol 19: 1821-1830, 1999.

33. Chou YT, Wang H, Chen Y, Danielpour D and Yang YC: Cited 2 modulates TGF-beta-mediated upregulation of MMP9. Oncogene 25: 5547-5560, 2006.

34. Singh G, Singh SK, König A, et al: Sequential activation of NFAT and c-Myc transcription factors mediates the TGF-beta switch from a suppressor to a promoter of cancer cell proliferation. J Biol Chem 285: 27241-27250, 2010.

35. Feng XH, Zhang Y, Wu RY and Derynck R: The tumor suppressor Smad4/DPC4 and transcriptional adaptor CBP/p300 are coactivators for Smad3 in TGF-beta-induced transcriptional activation. Genes Dev 12: 2153-2163, 1998.

36. Koinuma D, Shinozaki M, Nagano Y, et al: RB1CC1 protein positively regulates transforming growth factor-beta signaling through the modulation of Arkadia E3 ubiquitin ligase activity. J Biol Chem 286: 32502-32512, 2011.

37. Liao M, Zhang Y, Kang JH and Dufau ML: Coactivator function of positive cofactor 4 (PC4) in Spl-directed luteinizing hormone receptor (LHR) gene transcription. J Biol Chem 286: 7681-7691, 2011.

38. Frederick JP, Liberati NT, Waddell DS, Shi Y and Wang XF Transforming growth factor beta-mediated transcriptional repression of c-myc is dependent on direct binding of Smad3 to a novel repressive Smad binding element. Mol Cell Biol 24: 2546-2559, 2004. 